\title{
Knowledge Discovery in Smart City Digital Twins
}

\author{
Neda Mohammadi \\ Georgia Institute of Technology \\ nedam@gatech.edu
}

\author{
Ambuz Vimal \\ Georgia Institute of Technology \\ avimal@gatech.edu
}

\author{
John E. Taylor \\ Georgia Institute of Technology \\ jet@gatech.edu
}

\begin{abstract}
Despite the abundance of available urban data and the potential for reaching enhanced capabilities in the decision-making and management of city infrastructure, current data-driven approaches to knowledge discovery from city data often lack the capacity for collective data exploitation. Loosely defined data interpretation components, or disciplinary isolated interpretations of specific datasets make it easy to overlook necessary domain expertise, often resulting in speculative decision-making. Smart City Digital Twins are designed to overcome this barrier by integrating a more holistic analytics and visualization approach into the real-time knowledge discovery process from heterogeneous city data. Here, we present a spatiotemporal knowledge discovery framework for the collective exploitation of city data in smart city digital twins that incorporates both social and sensor data, and enables insights from human cognition. This is an initial step towards leveraging heterogeneous city data for digital twin-based decision-making.
\end{abstract}

\section{Introduction}

Prior to the big data revolution and emerging sources of urban data, researchers studied the dynamics of cities with relatively high levels of abstraction, mostly from a theoretical perspective. Recently, researchers have begun to shift their focus to the problem of making sense of the flood of high resolution spatiotemporal city data that is now available, linking it to growing urbanization issues. How we make sense of this new abundance of city data determines how we make decisions that will shape the future of cities and the quality of the life of their citizens. A city is generally studied from the perspective of its largest infrastructure elements and its sustainability deficit. While a city's infrastructure systems provide fundamental services such as water, energy, transport, and communication for its citizens, these services significantly impact sustainability. Research shows that all of the United Nations (UN) 2030 Agenda targets for 5 of its Sustainable Development Goals (SDGs 3, $6,7,9$, and 11) are influenced by urban infrastructure $[1,2]$, highlighting the importance of interdependencies among city infrastructure and their collective impact on urban sustainability. The availability of high resolution spatiotemporal sensor data for city infrastructure services is opening up important opportunities to take advantage of its unique potential to support enhanced decision-making and better management of cities towards sustainability. Moreover, acquiring spatiotemporal knowledge of events that take part in relation to urban infrastructure elements from social data (e.g., georeferenced data from social media and mobile applications) provides additional insights into city dynamics. The accurate exploitation and interpretation of these heterogeneous data will enable us to capitalize on smart city interventions that not only improve the sustainable operation of the city's infrastructure, but also take the needs and preferences of its citizens into consideration. However, current approaches to knowledge discovery from city data often fail to provide adequate capacity for collective data exploitation and are limited in their ability to effectively integrate the necessary domain expertise into data interpretation components. This indicates that, irrespective of its crucial role, domain expertise can be overlooked in the process of automation or disciplinary isolation. Here we discuss the current challenges facing knowledge discovery from city data and present a spatiotemporal knowledge discovery approach that better supports the collective exploitation of city data in smart city digital twins.

\section{Knowledge Discovery from City Data}

Effective knowledge discovery in cities utilizing the heterogeneous data collected from multiple sources is paramount in achieving smart city objectives. This data can be generated from sensors or social platforms, 
and may take the form of structured (i.e., defined and searchable data types) or unstructured data (e.g., audio, video, and social media postings) that are not as easily searchable, including untapped information that requires domain knowledge expertise to interpret. Current approaches to knowledge discovery from city data are either data-centric or focused on a particular use case application.

Data-centric approaches focus on the collection, processing, and management of city data regardless of its source or application [3, 4]. These approaches appear to have only a limited focus in establishing a final data interpretation component. Cheng et al. [3] built CiDAP, a city data and analytics platform that integrates both unstructured and structured data from human activities and Internet of Things (IoT) sensor devices to serve part of a smart city testbed. Although benefitting from a comprehensive system architecture, the final 'applications' layer of the system is only loosely defined, and there is little detail on how the final data made available through such complex data processing and analytics system can be utilized in practice. Similarly, the services \& applications layer in Snchez et al.'s [4] smart city platform appear to be disintegrated, branching out to loosely defined application-specific data queries at the final stage.

In contrast, application-centric approaches to city analytics tend to be overly focused on a particular end point and may thus overlook any indirect effects by excluding data sources $[5,6]$. For example, Jin et al.'s [5] IoT-based urban information system is heavily focused on the management and processing of the collected data, and is dispersed into disciplinary applications. Its contribution to data interpretation, which is the most critical step, is limited to suggesting user-understandable visualizations while acknowledging that it is "challenging to visualize heterogeneous sensory data". Application-centric approaches such as Lècuè et al.'s [6] STAR-CITY, a system built on human and machine-based sensor data to implement semantic traffic analytics (i.e., a focus on transportation), although application oriented and discipline specific, suffers from the similar limitations. Focusing on single infrastructure elements fail to take into account the interdependencies between different city infrastructure networks and can thus lead to misleading conclusions. Cities are complex evolving systems [7] and overlooking the complex interdependencies between humans, infrastructures, and technologies, along with sustainability concerns, may fail to support integrated management decisions. Khan et al. [8] has presented a more theoretical perspective on integrating information when dealing with sustainability and the socioeconomic growth of the city by collectively examining crime and safety/economy and employment data. Although his perspective is promising, it lacks appropriate empirical validation and does not incorporate human reasoning and domain expertise.

In this paper, we present a spatiotemporal knowledge discovery framework for smart city digital twins to explore the properties of an integrated approach to analytics and the representation of city data. We further demonstrate the application of this framework for an urban health knowledge discovery case. This framework, part of an ongoing project, draws on insights from human intuition and cognition to facilitate the collective discovery of knowledge from both social and sensor data.

\section{Knowledge Discovery in Digital Twins}

\subsection{Cities in Space and Across Time}

Complexity is a systemic property of cities [7]. Evolving complex systems are best understood as dynamic networks of interdependent interactions and relationships. Such interactions and relationships among humans, infrastructure, and technologies in cities [9], in space and across time, can not be regarded as mere aggregates of independent entities or analyzed independently. The advent of geographic information system (GIS) technology in the 1960s has made spatial data such as satellite imagery and aerial photography available to urban scientists and planners. Temporal sets of this spatial data enabled the early stages of spatiotemporal modeling of urban dynamics $[10,11,12]$. More recently, technological advances and the widespread usage of global positioning system (GPS) technology at the individual (e.g., smart phones, wearable devices) and infrastructure levels (e.g., IoT sensors and devices in buildings and transportation netwroks), now generates spatial data in significantly higher volumes and finer resolutions that is streamed at a real-time temporal resolution. Moreover, the ability to access social media platforms (e.g., Twitter, Instagram, Youtube, Facebook) on mobile devices has created a huge influx of georeferenced socio-economic data. Access to this level of high resolution spatiotemporal data in cities allows for more complex urban modeling and simulations, in which dynamic interactions and interdependencies can be captured in space and across time. However, meaningful knowledge discovery from such heterogeneous sources of urban data remains challenging for data abstraction and aggregation, and issues with noise and missing data are problematic when integrating heterogeneous datasets and linking 
structured to unstructured data. Simulating cities in virtual environments appears to be a promising avenue for facilitating these challenges $[9,13]$.

\subsection{Cities across Reality-Virtuality}

Creating virtual urban environments has traditionally been limited to static physical representations of the city $[14,15,16]$. Integrating these static physical representations of the city with dynamic spatiotemporal data, in which the reality-virtuality content coexists interactively can boost model accuracy in space and across time, greatly facilitating knowledge discovery. Continuously streaming sensor-based reality data into this (digital twin) environment, the static physical representation becomes smarter, and the spatiotemporal data is put into context.

Smart city digital twins [7] encompass Milgrams virtuality continuum [17] (Figure 1), which expands from complete real to complete virtual environments (i.e., Virtual Reality (VR)). Any combination of the two is located in-between, where real and virtual objects are combined in either real (i.e., Augmented Reality (AR)) or virtual surrounding environments. Similarly, Mixed Reality (MR) refers to the merging of both real and virtual environmnets, in which both real and virtual objects are accessible [17].

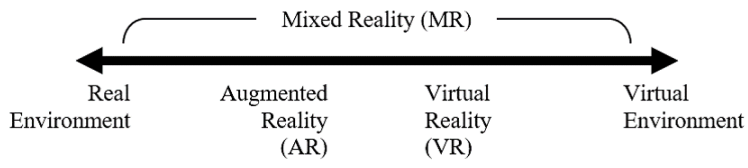

Figure 1. Virtuality continuum. Adapted from "A taxonomy of mixed reality visual displays" by Milgram and Kishino [17].

Current virtual city models often lack a coherent approach to knowledge discovery. They either consist of limited data-types simply visualized in the model without any analytics or simulation capabilities, such as CityHeat (heat related to traffic using color-mapped cubes) [18], VR Digital City Model (DCM) (landmark locations and buildings) [19], Connected Cities VR (city maintenance and 311 complaint information) [20], and AR City (navigation and route highlights); or are targeted towards particular audiences such as AR City (users walking through the city), Connected Cities VR (mayors and city stakeholders), and CityScapes AR (Tourists visiting NYC). Although representative, these virtual models are narrow in scope and lack the ability to provide realistic simulations in space and across time, incorporate interdependencies, and enable a collective knowledge discovery process for a wide range of disciplines and city stakeholders.

Both VR and AR technologies provide affordances that enable more collective interpretation of data and can enhance knowledge discovery. However, channeling this heterogeneous city data into appropriate interactive representations in VR and AR remains a challenge. In the following sections, we discuss the most prominent VR and AR affordances for virtual city modeling that can facilitate knowledge discovery. We then present the digital twin of an urban campus which allows for spatiotemporal knowledge discovery from both social and IoT sensor data for both outdoor (across a campus corridor) and indoor (inside campus buildings) environments while the users interact with both the physical and virtual infrastructures. It can analyze and track changes that take place in the real environment in space, across time, and within context. This has given rise to the areas of Immersive (using VR) [21] and Situated (using AR) Analytics [22, 23].

\subsection{Harnessing the Affordances of VR for Knowledge Discovery}

Unlike computer monitor displays, where data visualizations are based on gestalt perceptual principles [24] and visual elements are organized into groups based on proximity, similarity, symmetry, etc. the major benefits afforded by a VR immersive environment are developed from the theory of affordance from perceptual psychology [25]. Affordance theory suggests that humans perceive the environment in terms of its possibilities for action.

Gibson [26] introduced the term affordances to represent all the action possibilities with an environment in reference to both the environment and the human. He further argued that humans exist in relation to the environment as a system and in order to fully comprehend a human behavior, it is necessary to study the environment within which the behavior has taken place. Norman [27] further links the affordance of an object to the mental and perceptual capabilities of the user as well as their past knowledge and experiences. This suggests the significance of VR for incorporating human perception, reasoning and domain expertise into knowledge discovery. From the perspective of affordance, VR provides the user with an extended sensory experience. Not only aural, haptic and kinesthetic approaches to data exploration are enabled, but also the user will experience a sense of presence within the ebbs and flows of data due to its perceptual and interactive properties (e.g., spatial perception, shape recognition, color differentiation, movement detection, etc.) [17]. These affordances help the user develop 
better insights while interacting with the data.

Although affordance theory was developed in response to the physical world, Stuckey et al. [28] argues that immersing the user in a virtual environment that is organized and structured in the same manner as the real environment (e.g., urban or natural scenes) can allow the user to similarly take advantage of the sensory inputs they receive through their perceptions from the real environment through the presence of various objects, artifacts, and things. When immersed in the virtual environment, although the user is still physically present in the real environment, their sensory experiences (visual, aural and kinesthetic) are predominantly stimulated by synthetic content. Therefore, a parallel virtual version (i.e., digital twin) of the real urban environment systematically augmented with extended sensory inputs from both social and sensor data from the real environment can create an ideal knowledge discovery experience. It enables a person to take part in the data exploration process by incorporating their domain expertise, which otherwise cannot easily be automated.

For knowledge discovery in VR to be effective, it is important to design a data exploration process in which human reasoning and the domain expertise of the users are combined with the computational power, analytics capabilities, and speed of data processing. Immersive analytics is most useful when we are not certain what we are looking for: the end goals are vague and can be regarded as a hypothesis generation process for testing "what if" scenarios [29]. This is particularly the case when dealing with unstructured data, which are not readily subject to analytics. Immersive analytics augments computational analytics with human cognition and visual perception. "Our conscious perception is unified at any given moment" [30]. Coherence is one of the most unique characteristics of human multimodal perception (e.g., our capability to seamlessly integrate diverse information). It is thus critical that all the information is relatively distributed in space and across time, and that all sensory inputs (including real world social and sensor data) are spatiotemporally bound and collectively stimulate the user's perception at the exact same time and location. This adds to the importance of matching the scale and resolution of various information sources in space and across time.

\section{Design and Development of an Urban Campus Corridor Digital Twin}

In this section we discuss the design and development of a digital twin of an urban academic campus (Georgia Tech) corridor comprising three main components: a VR-based analytics platform, linked to an AR-based mobile application, coupled with a social media platform (Twitter) to enable spatiotemporal knowledge discovery from heterogeneous data. The VR-based analytics platform is built on the Unity cross-platform game engine [21]. The platform is directly linked multiple application programming interfaces (APIs) (e.g., AoT HTTP API) and web portals (e.g., Georgia Tech EnergyWatch) for real-time sensing of both outdoor and indoor environments. The system architecture includes an iterative loop, within which data, in different formats from various sources, are first regulated and spatiotemporally stamped (classified into various spatial and temporal scales based on their input resolutions), and then semantically stamped as they are immediately populated into one or more of the predefined city infrastructure performance areas (Figure 2). Once all the necessary links are established, these datasets are stored in a Structured Query Language (SQL) database linked to a heterogeneous data repository that subscribes to the platform for access and retrieval, where the data is browsable and each datapoint has a spatiotemporal as well as a semantic stamp and can be retrieved and called into the VR environment at the corresponding time and location.

\subsection{City Infrastructure Performance Areas}

The current meta-data includes data from four different sources: (1) indoor IoT sensors including data from building level electricity and gas meters; (2) outdoor Array of Things (AoT) sensor nodes including ambient data as well as urban dynamics (e.g., vehicle counts); (3) AR mobile application (CitySnap), including unstructured georeferenced images, text, and demographic data; and (4) social media (Twitter), including georeferenced postings. Table 1 shows the various sensors and sources that feed data into the system together with their SDG and city infrastructure performance classifications: energy, water, health, environment, mobility, safety, equity, and beauty. The process of semantic stamping is currently performed by allocating the source sensor to the corresponding performance area. Links between the data sources are then generated by time and space. This process can be automated if the number of sensors for the performance areas exceeds a certain limit.

\subsection{AR Crowdsensing Mobile App: CitySnap}

Citysnap is an iOS AR crowdsensing mobile application that is designed to capture citizen feedback 


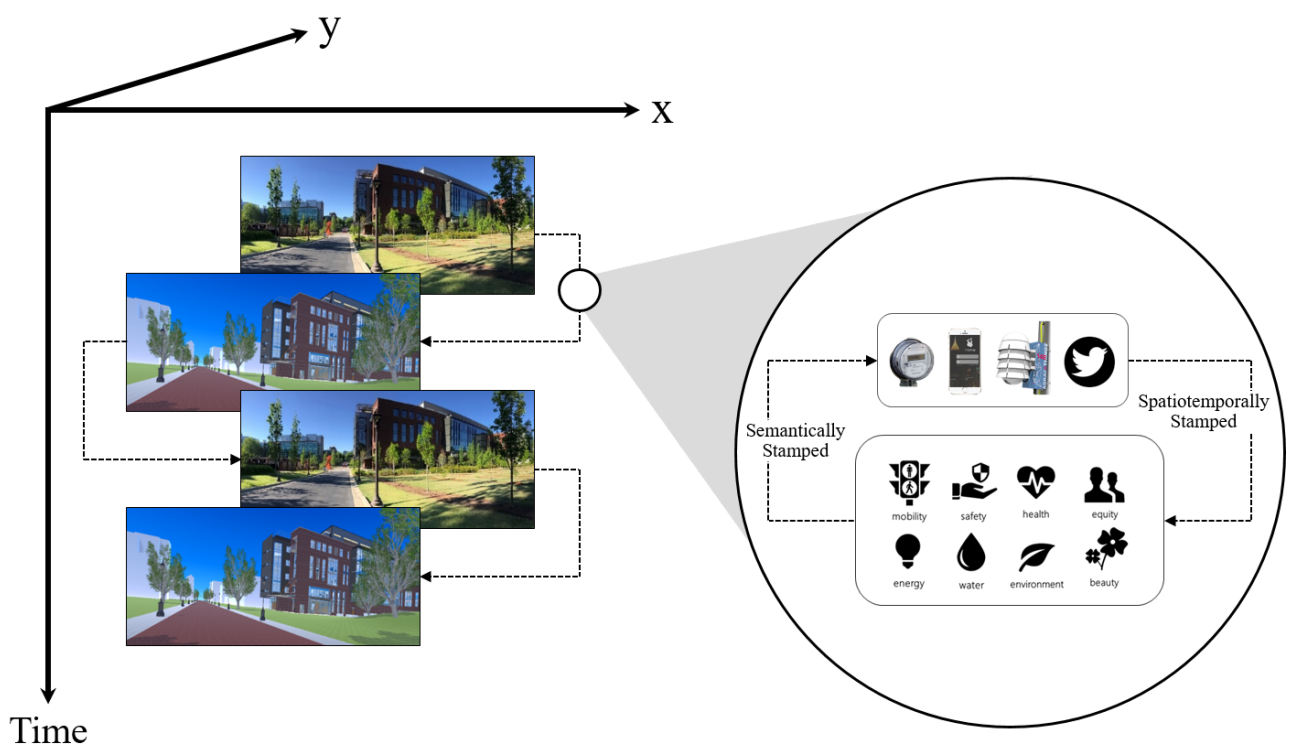

Figure 2. Spatiotemporal knowledge discovery: Graphical representation of Digital Twin data flow across time and space $(x, y)$; as the time progresses, the Digital Twin gets updated with new real-time data. Every data point is both spatiotemporally and semantically stamped and can be accessed and retrieved in the VR environment.

about the city infrastructure in relation to the predefined performance areas, while reciprocally presenting them with in situ information and interactivity that extends the physical world. Figure 3 depicts the interface of the application with an example of a user snapped image. By tagging the snapped image to a city infrastructure element linked to a performance area and including editable feedback comment, the user generates a georeferenced datapoint that appears directly in the co-located digital twin VR view. Similarly, any feedback virtually generated and tagged to the same infrastructure in the same location appears in the AR for the user to observe. Current and past postings, color-coded by performance area and visually attached to the infrastructure, are available both in a see-through AR view and a Map view for the user, where they can edit/remove the posting.

\subsection{Social Media Crowdsensing}

Information has a social life [16], on account of the fact that information is embedded in a social life and depends on context. Therefore, it is important to incorporate this data into the knowledge discovery process and make it available at the location where it is originally generated, making it more responsive to social and environmental settings. Visualizing social media data occurs in the co-located digital twin VR view [22]. Here, georeferenced social media data
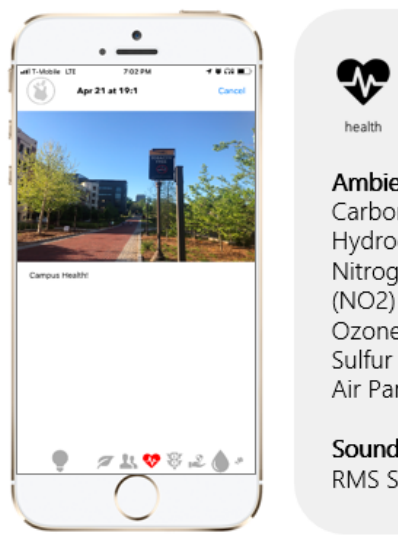

Ambient Air Quality

Carbon Monoxide Hydrogen Sulphide Nitrogen Dioxide

(NO2)

Ozone (O3)

Sulfur Dioxide (SO2)

Air Particles (PM)

Sound intensity RMS Sound Level

Figure 3. CitySnap: augmented reality crowdsensing mobile application.

is currently being anonymously collected through the public Twitter Stream API from users who voluntarily share georeferenced posting data.

\subsection{Interactive Knowledge Discovery in VR}

The current user interaction with the data in the digital twin is via a spatiotemporally sensitive on-demand HUD, which operates on the basis of a network of attached game objects and scripts. The spatiotemporal sensitivity of the HUD is set to update 
Table 1. Sensor/social city data per Infrastructure Performance Area in relation to the most impacted UN SDGs.

\begin{tabular}{|c|c|c|}
\hline Performance Area & UN SDG & Sensors \\
\hline Energy & 7 & Building-Level Electricity (HVAC, Elevators, Lighting, Café etc.); and Gas \\
\hline Water & 6 & Water, Chilled Water, Steam, Rain Water \\
\hline Health & 3 & $\begin{array}{l}\text { Ambient Air Quality (Carbon Monoxide, Hydrogen Sulphide, Nitrogen Dioxide, } \\
\text { Ozone, Sulfur Dioxide, Air Particles); and Sound Intensity (RMS Sound Level) }\end{array}$ \\
\hline Environment & 11 & $\begin{array}{l}\text { Weather Condition (Barometric Pressure, Humidity, Temperature); and Cloud Cover/ } \\
\text { Sunlight Intensity (Infrared Light, Light, Ultraviolet Intensity, Visible Light) }\end{array}$ \\
\hline $\begin{array}{r}\text { Mobility } \\
\text { Safety }\end{array}$ & 11 & $\begin{array}{l}\text { Street Conditions/Traffic flow/Events; and Detect Heavy Vehicles/Shock to Street } \\
\text { Pole (Magnetic Field, Acceleration and Orientation, Physical Shock/Vibration) }\end{array}$ \\
\hline $\begin{array}{l}\text { Equity } \\
\text { Beauty }\end{array}$ & 11 & $\begin{array}{l}\text { Citizen Feedback (CitySnap: Spatiotemporally Referenced Comments \& Images; and } \\
\text { Twitter: Georeferenced Social Media Postings) }\end{array}$ \\
\hline
\end{tabular}

as the data presented to the user changes by a set radius as the user navigates in the VR environment and only the spatiotemporally relevant data are collectively made available. The user can choose to see/filter the data by performance area and toggle between or overlay various data types within each performance area (e.g., Temperature and Humidity within the Environment performance area) as well as across difference performance areas (e.g., Temperature from Environment and Sulfur Dioxide (SO2) Air pollution from Health). Spatiotemporally and semantically linked user comments and tagged images from the corresponding CitySnap and social media postings appear in a side pane as the user navigates and interacts with the data. An interactive graph representation is coupled with a temporal slider that allows the user to navigate across time and choose to explore specific points or windows in time. The temporal range of the slider automatically matches the first and the last data entry. Any user-selected window in time can be separately viewed in a radar graph or as a pop out interactive 3D bar chart or cord diagram both indoors and outdoors in the VR environment (Figures 4-5). The user is thus able to customize and compare any two or more combination of data from any sensor. A number of typical interactive features are listed below:

AutoScroll - the graph view has an auto scroll functionality that allows the graph to temporally scroll through the data. The speed of the automatic scrolling can be controlled using the left hand controller's thumbstick.

Temporal Range - the temporal range of the graph data can be controlled using the right hand controller's thumbstick (pushing the thumbstick to the right increases the temporal range of the graph, thereby showing more graph points, while pushing the thumbstick to the left decreases the temporal range of the graph, thereby showing fewer graph points).

Go to-a particular instant in-Time - selecting a CitySnap or a social media posting brings the user to the same instant of time in the graph view when the posting has originally been generated by a user. Similarly, selecting any instant of time on the graph will populate all temporally associated postings generated by users.

Go to-a particular instant in-Space - selecting any CitySnap or social media posting (and using "Go to Location") teleports the user to the VR location that the posting has been generated, where a spatially associated (latitude, longitude, and compass direction) tweet panel or real image from the CitySnap is displayed.

These interactive features are designed to provide the user with sensory inputs from the environment that are spatiotemporally relevant, and semantically bound to facilitate a collective knowledge discovery experience in every space and across time. Virtually overlaying the environment with sensor and social data adds an additional layer of, otherwise unavailable, sensory inputs, which can help the user explore and exploit knowledge more effectively. Readily available correlations and spatiotemporal associations among various structured and unstructured datapoints enable the user to navigate the root cause of an anomaly and allow for an enhanced decision-making process.

\subsection{Urban Health Knowledge Discovery Case}

An urban health knowledge discovery case example described here demonstrates how the spatiotemporal knowledge discovery framework introduced in this paper links structured to unstructured data across the time and space dimensions and can enhance knowledge discovery from integrated heterogeneous sources of 


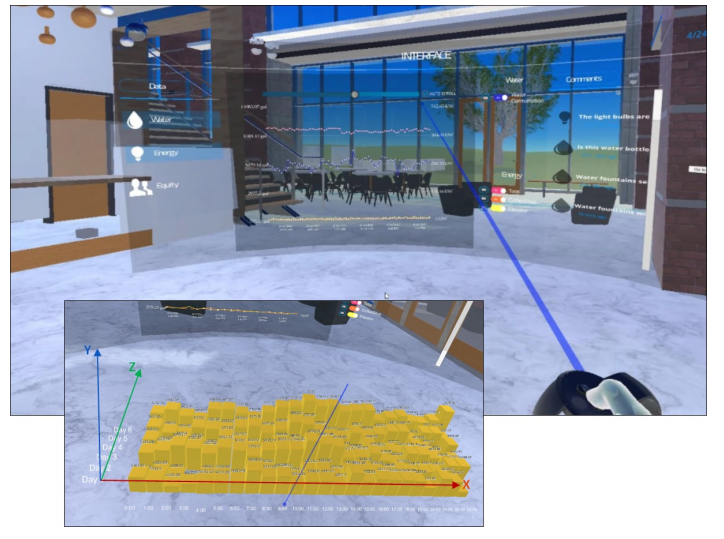

Figure 4. On-demand Digital Twin head-up display (HUD). Georgia Tech Urban Academic Campus (indoor). $\mathrm{X}$-axis indicates the time of the day; $\mathrm{Y}$-axis indicates the attribute value (e.g., Temperature in $\mathrm{C}$, Humidity in \%); Z-axis indicates the day of measurement.

urban data. Figure 6 depicts the process of data exploration in the urban campus corridor digital twin for a case of Health performance area (see Table 1). Considering various air quality measures listed in Table 1 as indicators of Health, at any given location in the VR campus corridor, the user can launch the head-up display (HUD) and select the Health icon from the left panel to display all semantically stamped available health-related data that belong to this specific location (Figure 6(a)); a Dynamic Radar Chart displays on the second panel from the left in the HUD depicting comparisons of multivariate air quality observations across time (Figure 6(b)); simultaneously, a Dynamic Time Series Graph of air quality data is displayed in the middle section, which can be navigated across time using the scrollbar. The user can overlay and toggle between various air quality measures to examine temporal fluctuations and identify spikes and anomalies (Figure 6(c)); for any combination of air quality measures, a 3D Cord Diagram can be displayed on-demand outside the HUD to explore the inter-relationships between various air quality measures. The darker and thicker the cord between any two air quality sensors, the stronger the correlation is between the two air quality measures (Figure 6(d)); finally, all the aforementioned graphs and data representations are spatiotemporally linked to unstructured health-related data in the right panel of the HUD, including user posted images from CitySnap (Figure 3), as well as postings from social media (Twitter) (Figure 6(e)). With a collection of structured and unstructured data at the user's finger tips, while immersed in a simulated virtual environment, the digital twin is providing the user

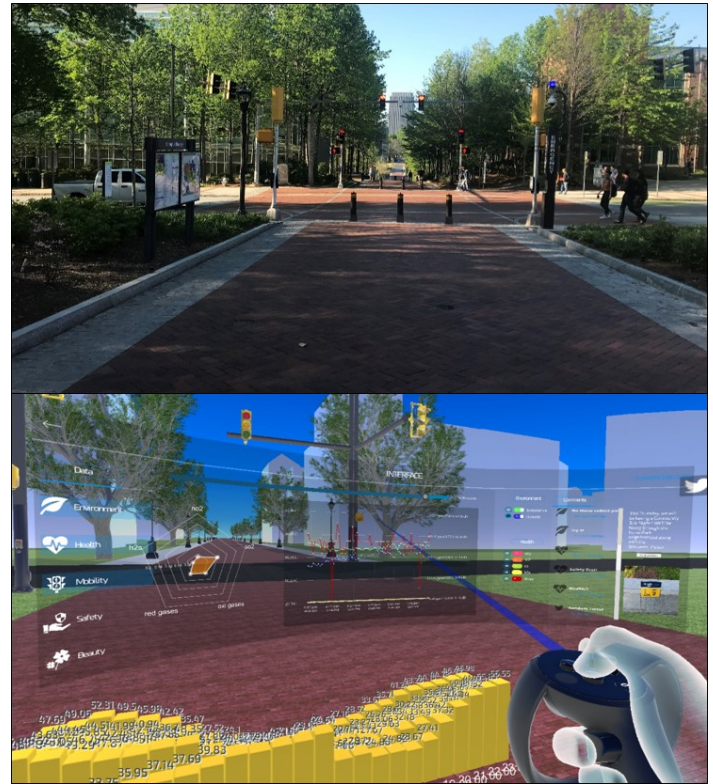

Figure 5. On-demand Digital Twin head-up display (HUD). Georgia Tech Urban Academic Campus (outdoor).

an with an extended sensory experience, incorporating human perception, reasoning and domain expertise into the knowledge discovery process.

Collective exploitation of city data in this way incorporates both social and sensor data, and enables insights from human cognition. This in turn promotes human reasoning and the domain expertise of the user, which is combined with the computational power, analytics capabilities, and speed of data processing at any given time and location in the VR environment. Integrating unstructured data, which are not readily subject to analytics, into the immersive analytics environment of the urban campus corridor digital twin facilitates hypothesis generation, identifying anomalies, synthesizing knowledge, and testing "what if" scenarios.

\section{IoT Interventions in Smart City Digital Twins and Future Directions}

A spatiotemporally data-rich digital twin such as the example described here, in which both fixed and mobile sensing of the infrastructure (via indoor and ambient sensors, crowdsensing mobile applications, and social media) enables the design and testing of various IoT interventions: (1) monitoring (historic and real-time) sensor data against thresholds to assess the current and ongoing conditions (i.e., what happens here?); (2) linking the observed correlations with the corresponding 

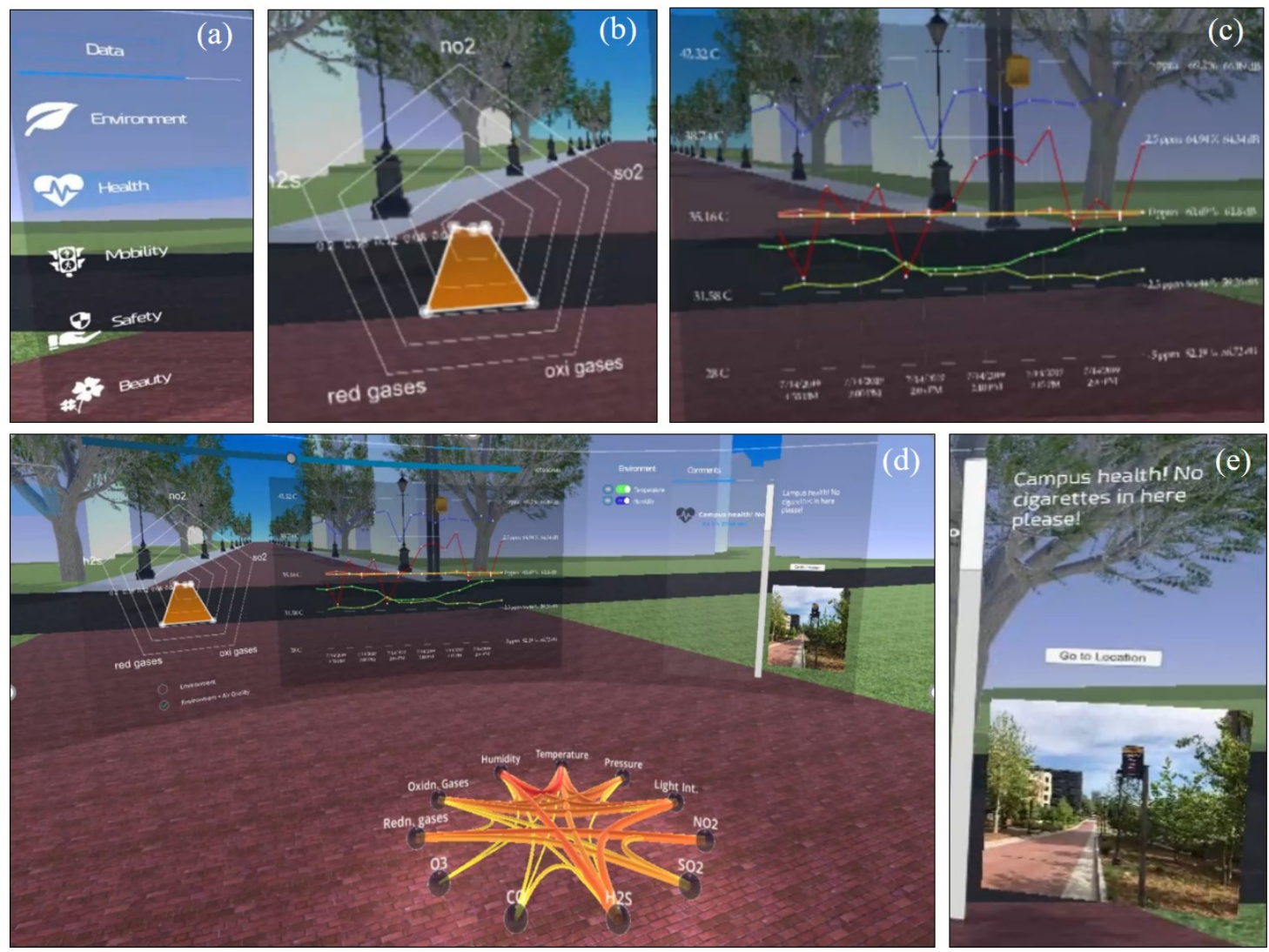

Figure 6. Urban Health Knowledge Discovery Case: (a) Health icon is selected from the left panel; a (b) Dynamic Radar Chart depicts comparisons of multivariate air quality observations across time; a (c) Dynamic Time Series Graph displays temporal fluctuations of air quality observations; an (d) on-demand Cord Diagram displays inter-relationships between various air quality measures; all are spatiotemporally linked to (e) health-related user posting images from CitySnap, as well as postings from social media (Twitter).

citizen feedback (i.e., data from CitySnap and Twitter) to determine the root causes of the problem (i.e., why it happens?); (3) perform predictions and time-to-event analyses to examine "what if" scenarios (i.e., what if - happens?); and finally (4) devise interventions to automate, optimize, and improve the current or ongoing conditions.

Future efforts will expand the two-way communication module of the digital twin such that it provides a more collaborative communication channel between city stakeholders (i.e., government, citizens, and the industry); reduce high levels of abstraction where only numerical values are considered (i.e., structured data) by more comprehensibly integrating unstructured data such that the user can explore the semantic interdependencies between and within performance areas, and allows for spatiotemporal event and anomaly detection (finding patterns in data and anticipating events that do not conform to expected behavior).

\section{Conclusion}

Historically, the dynamics of cities were understood with relatively high levels of abstraction from the perspective of its largest infrastructure and their significant impact on sustainability. More recently, high resolution spatiotemporal sensor data on city infrastructure services has brought the promise of enhanced decision-making and management for cities. However, current approaches to knowledge discovery from city data lack the capacity for collective data exploitation and effective integration of domain expertise. We discussed the current challenges in knowledge discovery from city data and presented a spatiotemporal knowledge discovery approach for collective exploitation of heterogeneous city data in smart city digital twins, which integrates VR, AR, and IoT technologies to create a sensory-rich data exploitation city environment for decision-makers. This is an initial step towards leveraging the full potential 
of these technologies in knowledge discovery from heterogeneous city data.

\section{Acknowledgement}

This study was supported in part by a research grant from the Georgia Tech Center for the Development and Application of Internet of Things Technologies (CDAIT). Any opinions, findings, and conclusions, or recommendations expressed in this material are those of the author(s) and do not necessarily reflect the views of the sponsoring agency.

\section{References}

[1] S. Thacker, D. Adshead, M. Fay, S. Hallegatte, M. Harvey, H. Meller, N. O'Regan, J. Rozenberg, G. Watkins, and J. W. Hall, "Infrastructure for sustainable development," Nature Sustainability, vol. 2, no. 4, pp. 324-331, 2019.

[2] UN DESA, "The Sustainable Development Goals Report 2018," tech. rep., New York, jul 2018.

[3] B. Cheng, S. Longo, F. Cirillo, M. Bauer, and E. Kovacs, "Building a Big Data Platform for Smart Cities: Experience and Lessons from Santander," in 2015 IEEE International Congress on Big Data, pp. 592-599, 2015.

[4] L. Sánchez, V. Gutiérrez, J. A. Galache, P. Sotres, J. R. Santana, J. Casanueva, and L. Muñoz, "SmartSantander: Experimentation and service provision in the smart city," in 2013 16th International Symposium on Wireless Personal Multimedia Communications (WPMC), pp. 1-6, 2013.

[5] J. Jin, J. Gubbi, S. Marusic, and M. Palaniswami, "An information framework for creating a smart city through Internet of Things," IEEE Internet of Things Journal, vol. 1, no. 2, pp. 112-121, 2014.

[6] F. Lécué, S. Tallevi-Diotallevi, J. Hayes, R. Tucker, V. Bicer, M. Sbodio, and P. Tommasi, "Smart traffic analytics in the semantic web with STAR-CITY: Scenarios, system and lessons learned in Dublin City," Journal of Web Semantics, vol. 27-28, pp. 26-33, 2014.

[7] M. Batty, The New Science of Cities. The MIT Press, 2013.

[8] Z. Khan, A. Anjum, and S. L. Kiani, "Cloud based big data analytics for smart future cities," in 2013 IEEE/ACM 6th International Conference on Utility and Cloud Computing, pp. 381-386, 2013.

[9] N. Mohammadi and J. E. Taylor, "Smart city digital twins," in The 2017 IEEE Symposium Series on Computational Intelligence (IEEE SSCI 2017), (Honolulu, Hawaii, USA), IEEE, 2017.

[10] T.-y. Shen, W.-d. Wang, H. O. U. Min, Z.-c. Guo, X. U. E. Ling, and K.-z. Yang, "Study on spatio-temporal system dynamic models of urban growth," Systems Engineering-Theory \& Practice, vol. 27, no. 1, pp. $10-17,2007$.

[11] M. A. Habib and E. J. Miller, "Influence of transportation access and market dynamics on property values: Multilevel spatiotemporal models of housing price," Transportation Research Record, vol. 2076, no. 1, pp. 183-191, 2008.
[12] G. Tian, Y. Ouyang, Q. Quan, and J. Wu, "Simulating spatiotemporal dynamics of urbanization with multi-agent systemsA case study of the Phoenix metropolitan region, USA," Ecological Modelling, vol. 222, no. 5, pp. 1129-1138, 2011.

[13] NRF, "Virtual Singapore," 2018.

[14] M. Batty, "Virtual reality in geographic information systems," The Handbook of Geographic Information Science. Oxford, Blackwell Publishing, pp. 317-334, 2008.

[15] M. Gibin, A. Singleton, R. Milton, P. Mateos, and P. Longley, "An exploratory cartographic visualisation of London through the Google Maps API," Applied Spatial Analysis and Policy, vol. 1, no. 2, pp. 85-97, 2008.

[16] A. Hudson-Smith, R. Milton, J. Dearden, and M. Batty, Virtual Cities: Digital Mirrors into a Recursive World. Centre for Advanced Spatial Analysis (UCL), 2007.

[17] P. Milgram and F. Kishino, "A taxonomy of mixed reality visual displays," IEICE Transactions on Information Systems, vol. E77-D, no. 12, pp. 1-15, 1994.

[18] V. Cristie, M. Berger, P. Bus, A. Kumar, and B. Klein, "CityHeat: visualizing cellular automata-based traffic heat in Unity3D," in SIGGRAPH Asia 2015 Visualization in High Performance Computing, p. 6, ACM, 2015.

[19] Bee Smart City, "Digital City Model (DCM)," 2017.

[20] A. Sedor, "Vive Debuts Future of VR City Planning," VR News, Games, And Reviews (Online), may 2017.

[21] T. Chandler, M. Cordeil, T. Czauderna, T. Dwyer, J. Glowacki, C. Goncu, M. Klapperstueck, K. Klein, K. Marriott, and F. Schreiber, "Immersive analytics," in 2015 Big Data Visual Analytics (BDVA), pp. 1-8, IEEE, 2015.

[22] N. ElSayed, B. Thomas, K. Marriott, J. Piantadosi, and R. Smith, "Situated analytics," in 2015 Big Data Visual Analytics (BDVA), pp. 1-8, IEEE, 2015.

[23] N. A. M. ElSayed, B. H. Thomas, K. Marriott, J. Piantadosi, and R. T. Smith, "Situated analytics: Demonstrating immersive analytical tools with augmented reality," Journal of Visual Languages \& Computing, vol. 36, pp. 13-23, 2016.

[24] K. Koffka, Principles of Gestalt Psychology. Routledge, 2013.

[25] J. J. Gibson, "The theory of affordances," in Preceiving, Acting, and Knowing, ch. 3, Hillsdale, NJ: Lawrence Erlbaum Associates, Publishers, 1977.

[26] J. J. Gibson, The Ecological Approach to Visual Perception: Classic Edition. Psychology Press, 2014.

[27] D. A. Norman, The Psychology of Everyday Things, vol. 5. Basic Books New York, 1988.

[28] S. U. Stucky, B. Shaw, and W. Ark, "Virtual environments overview," tech. rep., IBM Almaden Research Center, 2009.

[29] D. A. Keim, "Information visualization and visual data mining," IEEE transactions on Visualization and Computer Graphics, vol. 8, no. 1, pp. 1-8, 2002.

[30] S. Zmigrod and B. Hommel, "Feature integration across multimodal perception and action: a review," Multisensory research, vol. 26, no. 1-2, pp. 143-157, 2013. 